\title{
Intralesional Talimogene Laherparepvec Regimen
}

National Cancer Institute

\section{Source}

National Cancer Institute. Intralesional Talimogene Laherparepvec Regimen. NCI

Thesaurus. Code C160063.

A chemotherapy regimen consisting of intralesional talimogene laherparepvec that may be used in the treatment of cutaneous melanoma. 